\title{
Regards sur le Premier Mésolithique en Charente- Maritime : Fontbelle (Villars-les-Bois)
}

Sylvène Michel, François Blanchet et Jacques Blanchet

\section{OpenEdition}

Édition électronique

URL : http://journals.openedition.org/rao/771

DOI : 10.4000/rao.771

ISBN : 978-2-7535-1609-0

ISSN : $1775-3732$

\section{Éditeur}

Presses universitaires de Rennes

\section{Édition imprimée}

Date de publication : 31 décembre 2009

ISBN : 978-2-7535-1086-9

ISSN : 0767-709X

\section{Référence électronique}

Sylvène Michel, François Blanchet et Jacques Blanchet, « Regards sur le Premier Mésolithique en Charente-Maritime : Fontbelle (Villars-les-Bois) », Revue archéologique de l'Ouest [En ligne], 26 | 2009, mis en ligne le 31 décembre 2011, consulté le 03 décembre 2020. URL : http:// journals.openedition.org/rao/771 ; DOI : https://doi.org/10.4000/rao.771 


\title{
Regards sur le Premier Mésolithique en Charente-Maritime : Fontbelle (Villars-les-Bois)
}

\author{
Aspects of the Early Mesolithic in Charente-Maritime: Fontbelle (Villars-les-Bois)
}

\author{
Sylvène MicheL * \\ avec la collaboration de François Blanchet ${ }^{* *}$ et Jacques Blanchet ${ }^{* * *}$
}

\begin{abstract}
Résumé : Suite aux prospections de J. et F. Blanchet, une série de sondages a été réalisée à Fontbelle (Villars-les-Bois, Charente-Maritime) pour définir plus clairement l'occupation de ce site. L'étude de la série lithique a mis en évidence, sur des matériaux siliceux locaux, une production intégrée au sein d'une unique chaîne opératoire, les lamelles servant essentiellement à la confection d'armatures et plus particulièrement de triangles isocèles.
\end{abstract}

Abstract: Further to the land surveys of J. and F. Blanchet, excavations have been undertaken at Fontbelle (Villars-les-Bois, Charente-Maritime) to define more clearly the occupation of this site. The study of the lithic series provides, on local siliceous materials, a production integrated within a unique "chaîne opératoire", bladelets being essentially destined for armatures production, especially isosceles triangles.

Mots clés : Premier Mésolithique, Charente-Maritime, étude techno-économique, territoire stylistique.

Key words: Early Mesolithic, Charente-Maritime, techno-economic study, style territory.

\section{De La PROSPECTION AU SONDAGE : PRÉSENTATION DU SITE DE FonTBELLE}

Le site de Fontbelle ${ }^{1}$ (Villars-les-Bois, Charente-Maritime - fig. 1) a été découvert sur le versant sud d'une colline à proximité immédiate du Souillac en 1975, au cours d'une des nombreuses prospections de surface menées par F. et J. Blanchet sur les communes de Villars-les-Bois et Brizambourg. Ces opérations ont révélé une vingtaine de sites mésolithiques dont certains ont livré des collections primordiales pour la compréhension, dans l'ouest de la France, du Premier Mésolithique - ou industries à triangles par opposition aux carquois à trapèzes du Second Mésolithique (Costa et Marchand, 2006; Marchand, 2008). Malgré le Programme Collectif de Recherche Fin Würm/début Holocène sur le littoral charentais (1993-1998), ces séries n'ont été que globalement observées et rarement mentionnées (Joussaume, 1981; Favre, 1993, 2000; Blanchet, 2004; Michel, 2006); elles n'ont jamais été intégrées à une étude technologique et économique. Suite à l'examen du matériel lithique de Fontbelle, une série de sondages a été réalisée sur ce site au premier semestre 2008, permettant ainsi d'étoffer le corpus du Premier Mésolithique (9700-6900 cal B.C.; Cupillard et Richard, 1998) en Charente-Maritime.

* Doctorante; UMR 6566, CreAAH, Université de Rennes 1, campus de Beaulieu - 263 avenue du général Leclerc, 35042 Rennes Cedex.

** Dépôt de fouilles archéologiques - 13 rue de l'Hôtel-Dieu, 86000 Poitiers.

*** Dépôt de fouilles archéologiques - 122 cours Paul-Doumer, 17100 Saintes.

1. Site numéro 17470501 de la carte archéologique de France. Coordonnées Lambert (Zone II étendue) : X = 383.600, Y=2092.275. Parcelles cadastrales 245 et 266 de la section AL. 


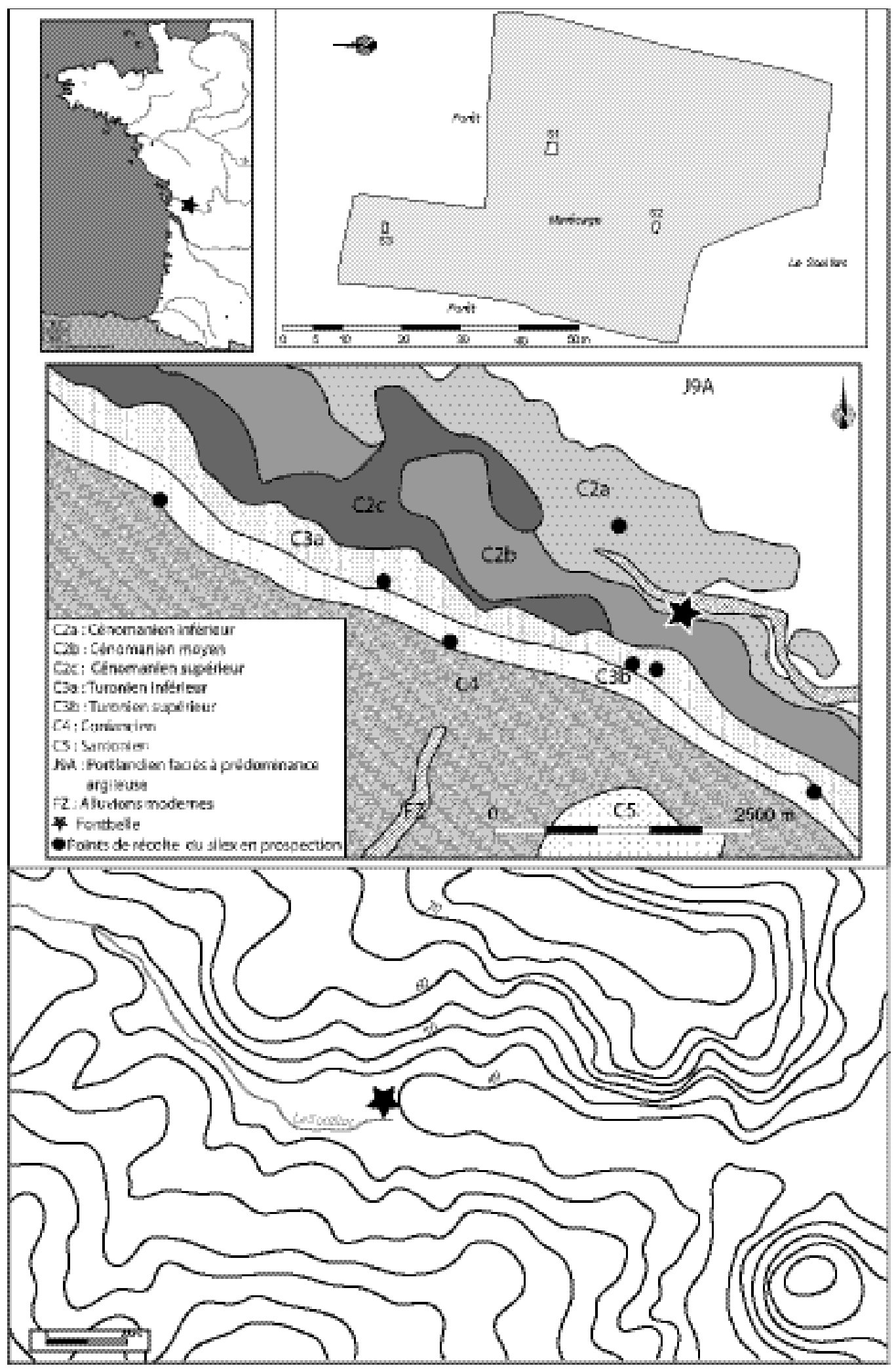

Figure 1 : Localisation de Fontbelle, implantation géologique et topographique (d'après les cartes du BRGM - feuille Saintes XV-31 à 1/50 000 - et de l'IGN - feuille Burie 1531-E à 1/25 000) et implantation des sondages dans la parcelle 245 .

Figure 1: Location of Fontbelle, geological and topographical position and location of excavations. 


\section{Un site, trois sondages}

Trois sondages de 2 à $4 \mathrm{~m}^{2}$ chacun, ont été réalisés (fig. 1). La couche de calcaire gréseux, appréhendée en S2, est apparue tronquée dans la partie supérieure de la parcelle suite à des phénomènes érosifs : ainsi, $S 1$ et $\mathrm{S} 3$ ne présentent plus, sur toute la hauteur explorée, que la couche argilo-sableuse sous-jacente (Cénomanien inférieur). Cette modification du terrain doit en partie être liée à la forte inclinaison des couches vers le sud-ouest, c'est-à-dire dans le sens de la pente actuelle. Le dénivelé entre les parties haute et basse de la parcelle est de $10 \mathrm{~m}$, soit une pente de $8 \%$.

Cet article ne traite que des résultats concernant S1, compte tenu :

- de l'indigence des indices au sein de S3, qui se trouve alors exclu de l'emprise de l'occupation mésolithique;

- de la position secondaire du matériel découvert en S2, au sein du labour et certainement associé à un léger colluvionnement;

- du fait que le matériel lithique de S2 et S3 n’apporte aucun élément nouveau aux conclusions que l'on peut émettre à partir de $S 1$.

Sous les terres labourées et jusqu'au fond du sondage à près d'un mètre sous la surface actuelle, le niveau sableux ne présente pas de réelles subdivisions mais seulement des variations de couleur allant du marron-orangé au beige, certainement dues à des migrations d'oxydes ferreux; aucune unité stratigraphique n'a donc été distinguée. Le tamisage des sédiments a été systématique afin d'obtenir un ensemble complet et représentatif. Outre l'occupation mésolithique, la présence de quelques scories dans le niveau labouré évoque des activités de sidérurgie ancienne et plus précisément la réduction de fer en bas fourneaux. Les tessons de céramique à glaçure verte également découverts dans ce niveau -81 fragments de très petites dimensions - rappellent ce qui est connu pour les $\mathrm{XIII}^{\mathrm{e}}$-XV ${ }^{\mathrm{e}}$ siècles en Centre-Ouest, attribution chronologique qui pourrait éventuellement concorder avec les activités métallurgiques.

\section{Limites taphonomiques et potentiel d'étude}

Un cinquième des pièces archéologiques mises au jour provient des terres brassées par les travaux aratoires. Les niveaux sous-jacents, eux, sont perturbés par des percolations liées à la nature du sol occupé : en S1, le mobilier lithique a été découvert dans des sables cénomaniens sur $60 \mathrm{~cm}$ de haut, sans que l'on y ait détecté de structures en creux. On note tout de même une chute brusque du taux de pièces à mihauteur (5,8 \% pour les trente centimètres inférieurs). Sur le site voisin des Prises (commune de Brizambourg), en milieu sédimentaire similaire, la série lithique se répartit sur $80 \mathrm{~cm}$ de haut (Blanchet et al., 2007). Ce type de phénomène taphonomique est courant sur les sites ouest-européens du Paléolithique final et du Mésolithique implantés en milieu sableux, qui ne présentent alors souvent plus de niveau(x) d'occupation mais des éléments dispersés sur 25 à $50 \mathrm{~cm}$ de haut suite à des bioturbations (Barton, 1987; Crombé, 1993; Vermeersch, 1976; 1995). Plusieurs hypothèses ont été formulées pour expliquer cette dispersion sur le plan vertical, avec l'activité biologique comme principal suspect. En plus des terriers de mammiferes, les petits agents fauniques jouent directement un rôle dans la dispersion verticale des éléments archéologiques : les lombrics, par exemple, permettent l'enfouissement des vestiges de $5 \mathrm{~mm}$ par an et auraient agit dès le début de l'Holocène en milieu forestier (Vermeersch, 1995).

Le degré d'homogénéité de l'assemblage peut être discuté à deux niveaux. Si l'on se place à l'échelle du Premier Mésolithique et de sa phase la plus ancienne telle qu'elle est définie par les modèles basés sur l'évolution morphologique des armatures depuis les travaux de J.-G. Rozoy (1978), l'ensemble est clairement cohérent avec de très rares intrusions d'occupations du Paléolithique final, du Mésolithique final et postérieures. On peut alors parler d'homogénéité relative pour une période qui s'étend tout de même sur plus d'un millénaire. Nous ne pouvons pas, par contre, affiner la résolution chronologique ou individualiser des occupations successives à Fontbelle, ce qui n'empêche pas d'y consolider les connaissances à l'échelle du Premier Mésolithique dans le Centre-Ouest.

\section{De L'ENTAME À L'OUTIL: ANALYSE DES INDICES D'OCCUPATION du Premier Mésolithique}

À l'ensemble récolté en prospection (1 225 éléments Blanchet, 2004) s'ajoutent désormais 9476 pièces lithiques dont $85 \%$ proviennent de $S 1$ (tableau 1). Elles ne sont malheureusement pas accompagnées de matériel osseux, l'acidité de la matrice sableuse encaissante n'ayant pas permis sa conservation. Les faibles surfaces ouvertes n'ont pas non plus livré de structure.

\section{Approvisionnement en matériaux siliceux}

Le matériel lithique est peu patiné, ce qui permet l'observation des matières premières employées qui s'avèrent être exclusivement du silex. Il revêt un aspect assez homogène, semi-opaque, aux variations de teinte allant du marron-beige au brun foncé. Ce silex correspond aux matériaux que nous avons pu récolter sur les affleurements turoniens dans les 


\begin{tabular}{|c|c|c|c|}
\hline \multicolumn{2}{|c|}{ Types de pièoes } & $\mathrm{N}$ & $\%$ \\
\hline \multirow{4}{*}{ Eclat } & entier & 437 & 12,1 \\
\hline & proximal & 30 & 0,8 \\
\hline & mésial & 35 & 1,0 \\
\hline & distal & 99 & 2,8 \\
\hline \multirow{4}{*}{ Lame } & entier & 91 & 2,5 \\
\hline & proximal & 193 & 5,4 \\
\hline & mésial & 176 & 4,9 \\
\hline & distal & 188 & 5.2 \\
\hline \multirow{4}{*}{ Lamelle } & entier & 113 & 3,1 \\
\hline & proximal & 473 & 13,1 \\
\hline & mésial & 513 & 14,3 \\
\hline & distal & 691 & 192 \\
\hline \multicolumn{2}{|c|}{ Nucleus } & 26 & 0.7 \\
\hline \multicolumn{2}{|c|}{ Bloc testé } & 3 & 0,1 \\
\hline \multicolumn{2}{|c|}{ Casson } & 157 & 4,4 \\
\hline \multicolumn{2}{|c|}{ Crête, néo-crête } & 12 & 0,3 \\
\hline \multicolumn{2}{|c|}{ Tablette } & 7 & 0,2 \\
\hline \multicolumn{2}{|c|}{ Outillage commun } & 23 & 0,6 \\
\hline \multicolumn{2}{|c|}{ Armature } & 151 & 4,2 \\
\hline \multicolumn{2}{|c|}{ Microbunin } & 178 & 4,9 \\
\hline \multicolumn{2}{|c|}{ Chute de burin } & 1 & 0,0 \\
\hline \multicolumn{2}{|c|}{ Sous-total } & 3597 & 100 \\
\hline \multicolumn{2}{|c|}{ Esquille de débitage } & 1754 & \\
\hline \multicolumn{2}{|c|}{ Esquille diverse } & 2704 & \\
\hline \multicolumn{2}{|c|}{ TOTAL } & 8055 & \\
\hline
\end{tabular}

Tableau 1 : Composition globale de la collection lithique de Fontbelle, en effectifs et en pourcentages.

Table 1: General count of the Fontbelle lithic assemblage: numbers and percentages.

$5 \mathrm{~km}$ alentours lors de prospections menées en 2008 (fig. 1). Ceci n'implique pas que les tailleurs se soient approvisionnés en ces points précis, la végétation du début de l'Holocène ayant pu plus ou moins masquer ses affleurements; on peut tout de même penser à un territoire d'acquisition des matières premières siliceuses taillables restreint. Au-delà du «territoire domestique élargi » (Geneste, 1992), il ne semble pas y avoir d'attrait pour le silex, à preuve la présence plus qu'anecdotique d'une seule pièce en silex d'Ecoyeux, pourtant disponible à quelque $7 \mathrm{~km}$ seulement. Ce choix est compréhensible compte tenu de la qualité des silex locaux qui assure une bonne conduite du débitage. Il évoque les stratégies déjà observées pour le Premier Mésolithique en Corrèze, Quercy et Pyrénées (Demars, 1998) ou encore à l'abri des Rocs (Bellefonds, Vienne: Fouéré in Joussaume, 1992; Gouraud in Joussaume, 1996).

\section{Du bloc originel au support}

Les indices récoltés évoquent une chaîne opératoire de production menée dans sa quasi-totalité sur le site même. En effet, si l'on se réfère aux pièces (semi-) corticales (11,3\% $\mathrm{du}$ corpus hors esquilles) et aux quelques blocs testés abandonnés immédiatement après l'apparition d'inclusions ou de plages mal silicifiées, on peut penser que les blocs de silex ont été, du moins en partie, introduits bruts sur le site. La mise en forme du bloc est simplifiée. Elle ne met pas en jeu de crêtes d'entame (à une exception près) et appelle plutôt un simple décorticage à l'aide d'un percuteur de pierre dure; les grands éclats ou lames alors extraits dégagent des nervures guides.

Que l'on prenne en compte les enlèvements bruts ou les négatifs lisibles sur les nucleus (fig. 2 et 3 ), le débitage est orienté vers la production lamellaire au sens large du terme. Hormis pour quelques nucleus à éclats dont l'organisation succincte ne permet pas une grande productivité, les productions de lames et d'éclats ne sont pas autonomes : elles interviennent au cours des processus visant à la fabrication de lamelles. Ces derniers produits ne sont ni réguliers ni standardisés et peu importe que l'enlèvement extrait soit strictement lamellaire ou approximativement allongé tel un éclat lamellaire. On peut s'interroger sur la nature de cette seconde catégorie de produits qui peut être tantôt d'origine intentionnelle, tantôt relever d'enlèvements strictement lamellaires qui ont rebroussé ou qui n’ont pas filé. La faible régularité des lamelles stricto sensu confirme que, même si l'on exclut les éclats lamellaires, les tailleurs de Fontbelle ne recherchaient pas des produits calibrés.

Lexploitation sur bloc laisse une place minoritaire au débitage sur éclat (fig. 3, $\mathrm{n}^{\circ}$ 3). La table s'implante alors dans l'épaisseur de l'éclat, son exploitation étant frontale à partir d'un seul plan de frappe, lisse, qui s'avère être la face inférieure de l'éclat-support. Pour les autres nucleus, le débitage se fait majoritairement sur une table, ce qui n'exclut pas parfois le recours à une seconde surface de débitage. Les tailleurs n'ont pas cherché à implanter la table dans une partie étroite du bloc ou à en conserver l'axe longitudinal pour le débitage. Les nucleus peuvent être exploités aussi bien de manière frontale (fig. 3, $n^{\circ} 1$ ) ou semi-tournante (fig. $2, n^{\circ} 3$, fig. 3, $n^{\circ} 2$ ) que sur la totalité de leur pourtour (exploitation tournante : fig. 2, no 1-2). Le débitage est essentiellement unipolaire. En présence d'un second plan de frappe en face opposée au premier, soit les séquences obtenues depuis l'un et l'autre sont clairement indépendantes et successives, soit la production est contrôlée par un plan de frappe, le second servant au contrôle de la carène. Les tailleurs ne préparent que sommairement les plans de frappe, les laissant lisses sans aménagement supplémentaire hormis l'abrasion de la corniche. Durant les séquen- 


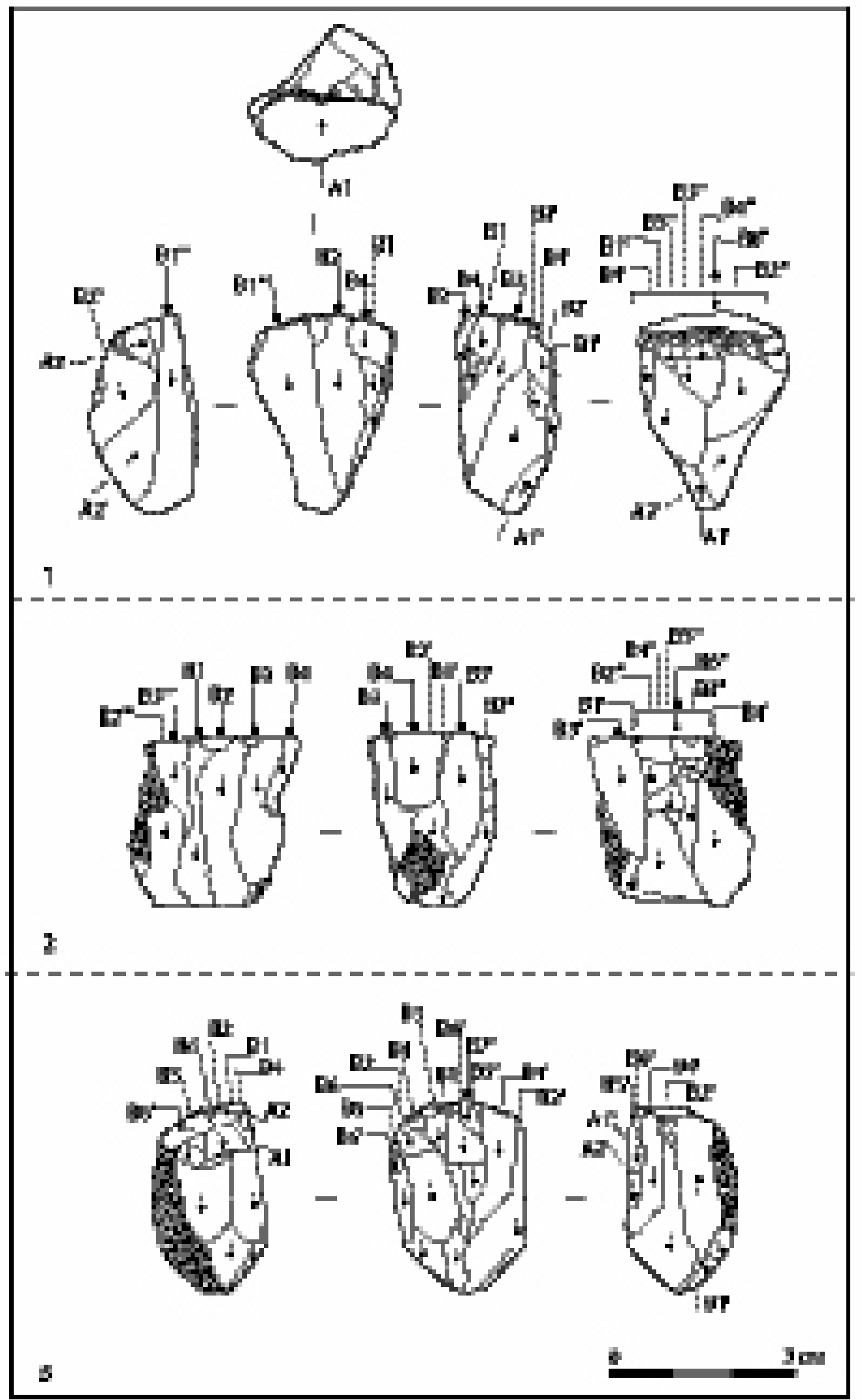

Figure 2 : Nucleus issus du sondage $1:$ nucleus unipolaires d'exploitation (semi-) tournante. Figure 2: Cores from Trench 1: unipolar cores with (semi-) rotating exploitation. 


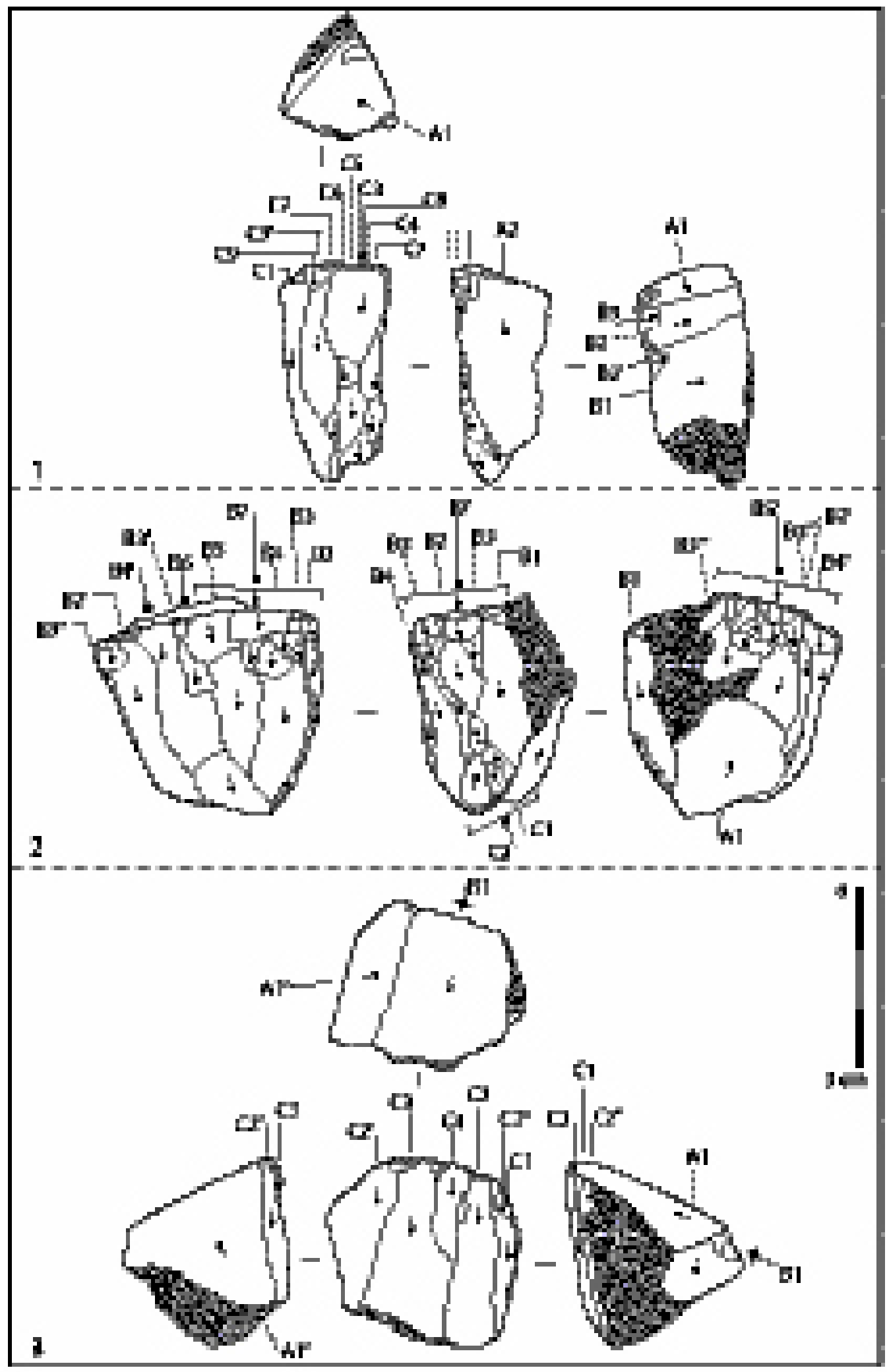

Figure 3 : Nucleus issus du sondage 1(suite) : 1, nucleus unipolaire d'exploitation frontale; 2 , nucleus semi-tournant à plan de frappe secondaire 3 , nucleus unipolaire sur éclat.

Figure 3: Cores from Trench 1 (continued): 1, unipolar with frontal exploitation ; 2, semi-rotating with secondary striking surface; 3, unipolar on flake. 
ces de débitage lamellaire, le tailleur a recours à un percuteur de pierre utilisé en percussion directe. En suivant les critères de J. Pelegrin (2000), les stigmates lisibles sur ces talons n'aident pas toujours à trancher quant à la dureté du minéral utilisé, même si les arguments vont plus dans le sens d'un percuteur de pierre tendre : des talons abrasés lisses minces, de quelques millimètres à punctiformes, des angles de chasse compris entre 70 et $80^{\circ}$, la récurrence d'une ligne postérieure irrégulière du talon, ainsi que la présence de petits bulbes avec identification ponctuelle d'esquillement du bulbe.

Les flancs, quand ils n'ont pas été investis par le débitage, ont été conservés corticaux ou ont participé à l'aménagement du volume avec le retrait, à partir du plan de frappe contrôlant la table, d'enlèvements débordants tirés parfois bien en arrière du bord pour reconquérir du cintre. Le dos se résume le plus souvent à une plage corticale mais n'est pas toujours exclu de la logique volumétrique comme le prouvent quelques exemplaires à crête postérieure. Les néo-crêtes, essentiellement partielles à un pan, sont peu fréquentes dans la série. Il en est de même pour les enlèvements de réaménagement du plan de frappe qui prennent la forme d'éclats, parfois semi-corticaux, tirés sans exception depuis la table lamellaire. Leur épaisseur montre que les tailleurs ne se préoccupaient pas du coût en matière première de cette opération, ce qui est compréhensible compte tenu des facilités d'approvisionnement.

L'abandon du nucleus répond à deux causes complémentaires : la présence de réfléchissements et un aplatissement marqué des convexités qui compromet le débitage. La perte de carène semble un problème majeur dans ces débitages, sans que les tailleurs y remédient pour autant : le changement de bloc est-il préféré aux réaménagements des blocs endommagés? Est-ce alors en lien avec la disponibilité locale du silex?

\section{Les armatures}

Les 151 armatures mises au jour $-4,2 \%$ du corpus hors esquilles; $86,8 \%$ de l'outillage - permettent une bonne approche de la préparation des activités cynégétiques (schéma opératoire de fabrication des armatures) et des choix stylistiques préférentiels (types et caractéristiques des armatures) (fig. 4; tableau 2). Le tamisage systématique des sédiments a notamment permis d'orienter le débat sur les liens entre les groupes de Poitou-Charentes et le techno-complexe méridional sauveterrien caractérisé par des armatures hypermicrolithiques dites triangle de Montclus ${ }^{2}$ et pointe de Sauveterre ${ }^{3}$.

2. Triangle au troisième côté retouché suivant une inclinaison semiabrupte à abrupte, élancé ( $\mathrm{L}>4 \mathrm{l}$ ) avec une grande troncature trois fois supérieure à la petite.

3. Pointe fusiforme de silhouette élancée $(\mathrm{L}>4$ l) et dessinée par deux bords abattus.

\begin{tabular}{|c|c|c|}
\hline $\begin{array}{c}\text { Types } \\
\text { d'ermatures }\end{array}$ & $N$ & $\%$ \\
\hline Triangle isocèle & 49 & 55,7 \\
\hline Triangle scalene & 14 & 15,9 \\
\hline Lamelle scalene & 1 & 1.1 \\
\hline $\begin{array}{l}\text { Pointe à base } \\
\text { retouchèe }\end{array}$ & g & 10,2 \\
\hline $\begin{array}{c}\text { Pointe a base } \\
\text { naturelle }\end{array}$ & 11 & 12,5 \\
\hline Segment & $\overline{9}$ & 3,4 \\
\hline Trepezze & 1 & 1,1 \\
\hline Total & $8 \theta$ & 100 \\
\hline $\begin{array}{l}\text { Armatura en } \\
\text { cours de } \\
\text { fabrication }\end{array}$ & 15 & \\
\hline $\begin{array}{c}\text { Fragment } \\
\text { d'armature (1) }\end{array}$ & 48 & \\
\hline TOTAL & 151 & \\
\hline
\end{tabular}

Tableau 2 : Effectifs et pourcentages des types d'armatures. (1) : pièces sans attribution typologique possible.

Table 2: Armatures: numbers and percentages of the different types. (1): pieces of no precise typological attribution.

\section{Du support au projectile : fabrication de l'armature}

Le mobilier contient 178 pièces attestant du procédé du microburin, à savoir des microburins à proprement parler et des lamelles à cassure dans ou au-dessus de la coche. Parmi leurs caractères principaux s'affichent une implantation préférentielle des coches à droite ainsi que la prédominance des éléments proximaux sur les distaux, ce qui s'explique certainement par la difficulté à modifier par la seule retouche la partie proximale des supports à cause du bulbe et du talon. Certains microburins ont conservé du cortex, avec d'ailleurs parfois une face supérieure entièrement corticale, ce qui souligne une certaine flexibilité dans la sélection des supports. La régularité des 178 pièces est d'ailleurs faible à moyenne et se couple à des profils légèrement tors. Notons pour finir la forte amplitude de la largeur de ces éléments (2,4 à $15,1 \mathrm{~mm})$.

Quatorze pièces possèdent un bord abattu sinueux dont part une cassure orthogonale à l'axe du support. Elle se résume dans la quasi-totalité des cas à des accidents de type Krukowski (Tixier, 1963). Si l'on considère ces pièces en cours d'aménagement, on peut alors penser que l'abattage du bord s'effectue en deux temps : amorce par retouches abruptes à semi-abruptes et finition par retouches abruptes régulières. Le côté opposé est conservé brut, ce qui placerait l'opération de bordage en fin de fabrication. 


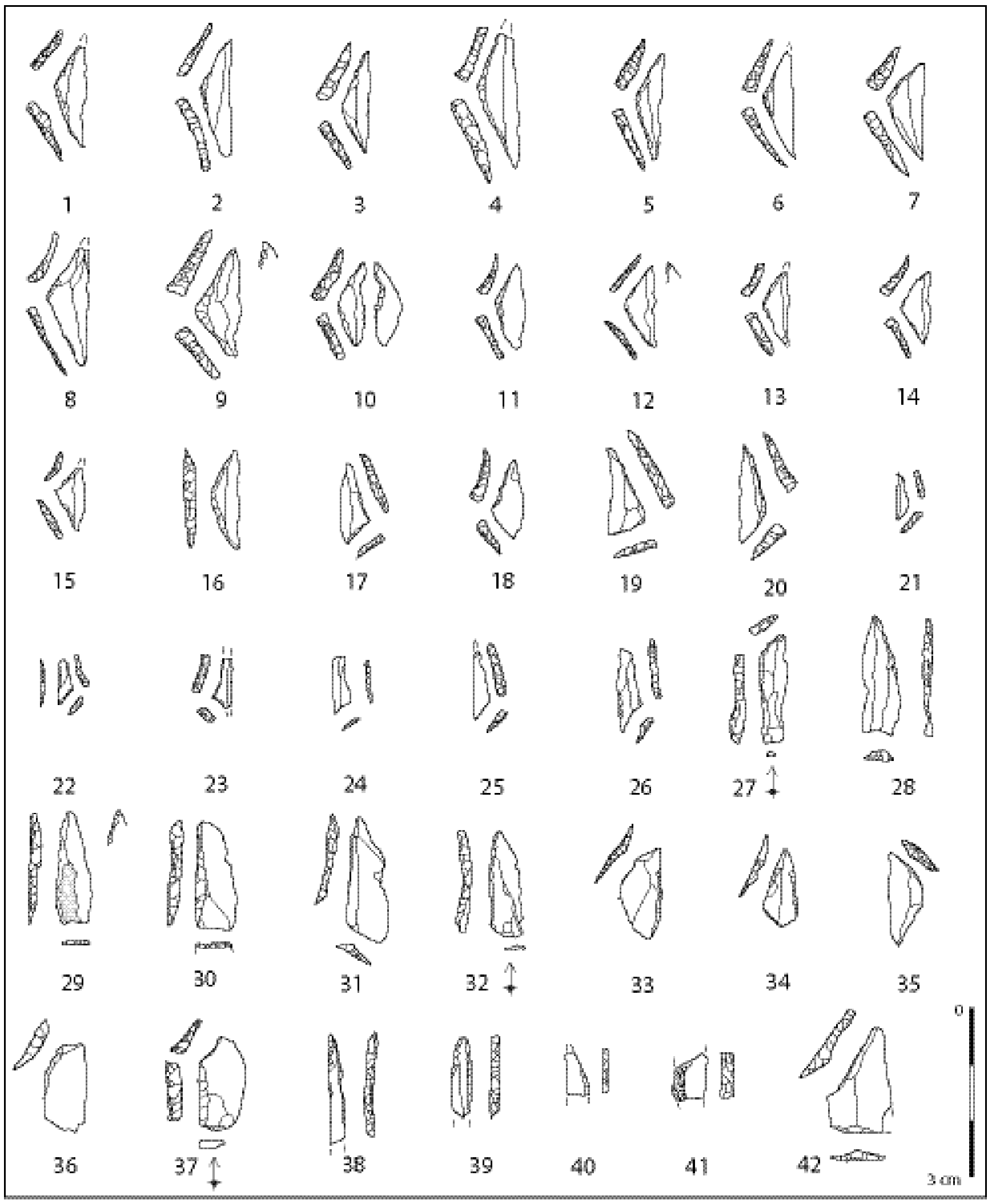

Figure 4 : Armatures issues du sondage $1: 1-15$, triangles isocèles; 16, segment; 17-26, triangles scalènes; 27, lamelle-scalène; 28-31 pointes à base naturelle; 32-37, pointes à base retouchée; 38-41, fragments; 42, trapèze rectangle.

Figure 4: Armatures from Trench 1: 1-15, isosceles triangles; 16, segment; 17-26, scalene triangles; 27, scalene-bladelet; 28-31, points with natural base; 32-37, points with retouched base; 38-41, fragments; 42, trapeze. 


\section{L'armature, de l'arme au marqueur socioculturel}

Les triangles, avec près des trois quarts de l'effectif, ne laissent que peu place aux autres types d'armatures. Une attention particulière a été portée aux modèles isocèles puisqu'ils forment plus de la moitié des armatures ayant obtenu une attribution typologique. Ces armatures ont dans un tiers des cas un profil tors, ce qui, plus qu'un trait recherché, traduirait une conséquence technique assumée. La conservation de cortex sur certains exemplaires portant des traces d'impact souligne la flexibilité des normes régissant les séquences de production et la sélection des supports. Quelques piquantstrièdres s'affichent en pointe (fig. $4, n^{\circ} 8$ ), d'autres ayant dû être entièrement retouchés. Aux retouches directes abruptes se substituent parfois des retouches croisées (fig. $4, \mathrm{n}^{\circ} 3$ ). Les troncatures suivent une délinéation rectiligne; quand elles sont légèrement concaves ou convexes, la morphologie générale de la pièce ne sort pas réellement du lot. Le troisième côté n'est que peu fréquemment bordé; parfois quelques retouches inverses semi-abruptes servent à appointer un peu plus l'armature (fig. $4, \mathrm{n}^{\circ} 10$ ). Les modèles longs, outre le fait d'être majoritaires dans ce corpus, sont en général légèrement plus étroits que les courts avec des largeurs moyennes respectives de 5,5 et $6,2 \mathrm{~mm}$.

Si l'on s'intéresse désormais aux triangles scalènes, on distingue les pièces proches du triangle isocèle (fig. $4, n^{\circ} 18$ ) des triangles scalènes allongés qui sont alors des hypermicrolithes (fig. 4, $\mathrm{n}^{\circ}$ 21-24). Dans ce second cas, la " distance de dégagement de la barbelure ", pour suivre la terminologie d'A. Thévenin (2005; fig. 5), peut ne mesurer qu'un douzième de la totalité de l'armature. Certaines possèdent un troisième côté bordé, mais une seule mérite l'assimilation au sous-type triangle de Montclus (fig. $4, \mathrm{n}^{\circ} 22$ ). Si l'on revient à l'ensemble des triangles scalènes, un des faits marquants concerne leur latéralisation quasi-exclusivement dextre. L'allure la plus récurrente pour les deux troncatures correspond à une délinéation rectiligne pouvant parfois tendre vers la concavité ou la convexité sans être souvent affirmée. Notons, pour la suite du raisonnement, la présence aussi bien des modèles $\left[\mathbf{a}=4-6 \mathrm{~mm} ; \alpha>115^{\circ}\right]$ et $\left[\mathbf{a}=7-12 \mathrm{~mm} ; \alpha<115^{\circ}\right]$ définis par A. Thévenin (ibid.; cf. infra). Une armature, avec un bord abattu rectiligne et une troncature en partie distale du support formant un angle de $45^{\circ}$ par rapport à l'orthogonale de la pièce, a été classée en lamelle-scalène (fig. $4, n^{\circ} 27$ ). Ce microlithe, qui a conservé un talon lisse mince, rappelle certains triangles scalènes mais aucun des deux modèles présents à Fontbelle, dont il se distingue nettement.

L'étude a révélé que près d'un quart seulement du carquois s'exprimait sous la forme de pointes, sans préférence marquée pour les modèles à base naturelle ou retouchée.

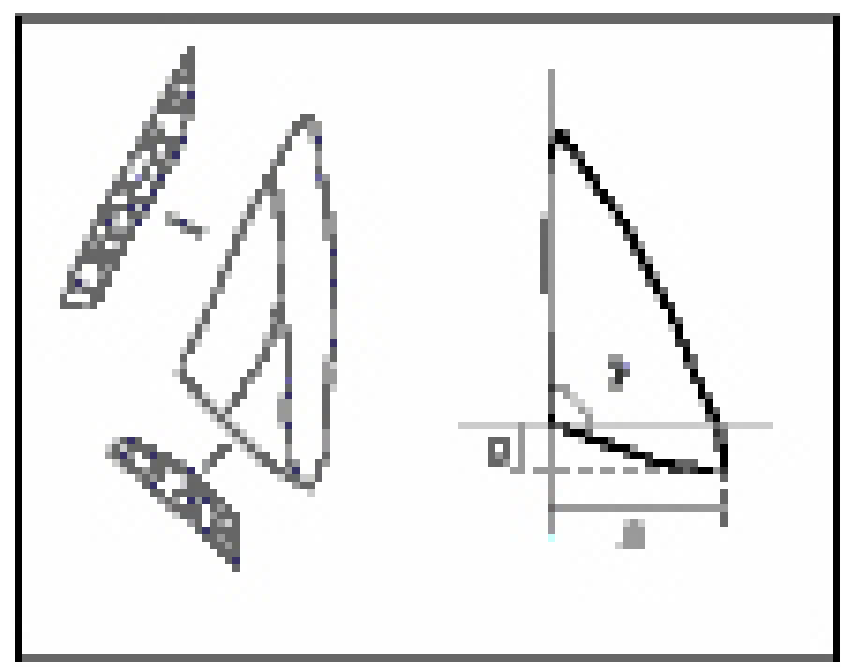

Figure 5: Schéma explicatif des mesures des triangles scalènes (d'après A. Thévenin, 2005).

Figure 5: Explanatory schema of scalene triangle measurments.

On peut en quelques points résumer ce qui les caractérise principalement :

- une latéralisation majoritairement senestre;

- le choix de placer la pointe du microlithe en partie proximale du support;

- le recours ponctuel à la retouche inverse rasante (fig. 4, $\left.\mathrm{n}^{\circ} 30\right)$;

- la variation de l'angle formé entre la troncature et l'orthogonale à l'axe du microlithe qui est soit proche de $45^{\circ}$, soit de $80^{\circ}$ dans le cas des troncatures très oblique;

- la rareté du bordage du côté opposé (fig. 4, n 33-34);

- la fréquente orthogonalité de la base lorsqu'elle est retouchée, avec alors une possible délinéation aussi bien rectiligne que concave (fig. 4, $\mathrm{n}^{\circ} 28-29$ );

- l'absence de pointes segmentiformes ou fusiformes, dont les sous-types de Chaville, de la Majoire et de Sauveterre.

Quelques pièces isolées semblent se démarquer de ces armatures attribuables au Premier Mésolithique : outre deux fragments épais de pointes à bord abattu, une monopointe évoque aussi une phase récente de l'Azilien.

Les segments, d'étroites pièces peu épaisses et symétriques, ne sont qu'au nombre de trois dans cet ensemble microlithique (fig. $4, \mathrm{n}^{\circ} 16$ ) ; l'un ressemble à certains triangles isocèles, le schéma morpho-technique pouvant éventuellement être similaire.

Avant même d'aborder la question de la fragmentation des pièces à l'utilisation, il est important de prendre en compte les armatures classées comme fragments dans cette partie. En effet, la plupart d'entre elles montrent un bord abattu, ce qui pourrait potentiellement les placer dans la catégorie des 
lamelles à bord abattu mais aussi, selon les cas, dans celles des triangles effilés ou des pointes.

Même si cela relève de l'anecdote, finissons ce tour d'horizon du fonds microlithique par la présence isolée d'un trapèze (fig. $4, \mathrm{n}^{\circ}$ 42). De nombreuses séries du Premier Mésolithique, y compris dans des contextes considérés à juste titre comme homogènes pour ne prendre que l'exemple de la couche 6 de Fontfaurès (Lentillac-Lauzac, Lot : Barbaza et al., 1991), ont livré un voire quelques trapèzes. Volonté originale, armatures inachevées, intrusions ponctuelles de matériel provenant d'occupations ultérieures, le sujet est à creuser. Notons en tout cas que ce modèle - trapèze rectangle à grande troncature concave latéralisé à gauche - traduit plutôt une phase précoce du Second Mésolithique et donc peut-être une phase transitionnelle entre les industries à triangles et le Mésolithique aux armatures dites évoluées.

\section{Suivre à la loupe des traces d'utilisation}

Les traces de macro-utilisation sont plus nombreuses sur les triangles scalènes et les pointes à base naturelle que sur les triangles isocèles et les pointes à base retouchée. Plus des deux tiers des fractures sont nettes, mais l'on distingue également des fractures diagnostiques de l'impact - en charnière, plume ou marche (Fischer et al., 1984) - qui ampute plus ou moins les armatures au niveau de leur apex (fig. 6). S’y ajoutent des cassures secondaires comme les spin-off (ibid.) et les coups de burin. Les triangles portent également des ébréchures sur leur troisième côté, tranchant ou retouché. En associant l'observation des stigmates (types, localisation, orientation...), on peut facilement joindre les pointes à base naturelle ou aménagée au groupe des pointes axiales (fig. 6). La détermination se complique pour les triangles : il semble que l'on ait plus fréquemment affaire à des barbelures mais certains scalènes, avec une intensification de l'abattage $\mathrm{du}$ bord vers la grande pointe, pourraient fonctionner en pointes barbelures telles qu'elles ont pu être définies dans le techno-complexe sauveterrien (Philibert, 2002; Chesnaux, 2008). Ces pièces combinent alors les fonctions de pointes axiales perçantes et de barbelures dilacérantes. Sans pouvoir interpréter cette donnée et la traduire en termes d'emmanchement, notons que la troncature des triangles qui porte l'impact est constituée de retouches souvent plus abruptes que la seconde.

\section{Un outillage commun des plus succincts}

L'outillage commun n'est constitué que de 23 pièces (fig. 7). Elles se rattachent majoritairement aux outils a posteriori et outils retouchés sans organisation de l'aménagement, qui forment 58,6\% de l'ensemble $\left(\mathrm{n}^{\circ} 9-12\right)$. Les supports alors majoritairement utilisés, des pièces épaisses conservant en partie des plages corticales, proviennent des phases de mise en forme et des séquences de réaménagement. Cette stratégie signe plus un choix délibéré qu'une contrainte liée au matériau, compte tenu de son abondance locale. Les outils aménagés sont peu diversifiés puisque la moitié d'entre eux sont des grattoirs ( $\left.\mathrm{n}^{\circ} 7-8\right)$. Les quelques outils encochés $\left(n^{\circ} 3-6\right)$, réalisés sur enlèvements de toute nature, ne voient leur bord modifié que par une seule coche. Leur statut de pièce achevée prête à l'emploi en tant qu'outil reste à confirmer; malheureusement, le sédiment encaissant n'est pas favorable à l'observation des traces de micro-utilisation (polis, émoussés, stries). La même question peut se poser pour les lames tronquées $\left(\mathrm{n}^{\circ} 1-2\right)$. On trouve également de manière isolée les types burin et couteau. S'y ajoute un nucleus dont les stigmates de réutilisation pourraient évoquer une réfection de percuteur. Aucun couteau à encoches basilaires n'est venu s'ajouter aux deux exemplaires découverts en prospections.

\section{Fontbelle, OU COMMENT EN SAVOIR un peu plus sur le Premier Mésolithique en Charente-Maritime?}

Nous avons ici affaire à une composante cohérente de pièces culturellement diagnostiques du Premier Mésolithique et plus exactement de sa phase ancienne $(9700-8020 \mathrm{cal}$ B.C. : Cupillard et Richard, 1998) si l'on s'en tient à la forte proportion de triangles isocèles. Les deux couteaux à encoches basilaires iraient dans le même sens puisque ce type d'outil assez caractéristique est associé à des corpus contemporains; une datation dans le dernier quart du $9^{\mathrm{e}}$ millénaire avant notre ère a d'ailleurs été obtenue à partir d'ossements humains d'une tombe de la Grande Pièce (La Vergne, Charente-Maritime : Duday et Courtaud, 1998; Courtaud et al., 1999) présentant huit de ces outils. À Fontbelle, une poignée d'armatures évoque une phase azilienne, ce qui n'est pas le cas des autres éléments lithiques : ainsi les nucleus - et leurs objectifs de production - ne traduisent rien de ce qui a été observé pour cette époque sur le site voisin des Prises (Brizambourg, Charente-Maritime : Naudinot in Blanchet et al., 2007). Cette composante reste anecdotique et nous pouvons ainsi proposer une esquisse des savoir-faire et des choix stylistiques concernant l'occupation mésolithique.

\section{Reconstitution des stratégies opératoires et questions de savoir-faire}

À Fontbelle, la production semble « intégrée au sein d'une unique chaîne opératoire " (Perlès, 1991) et orientée pour fournir des supports d'armatures. Les sous-produits, eux, 


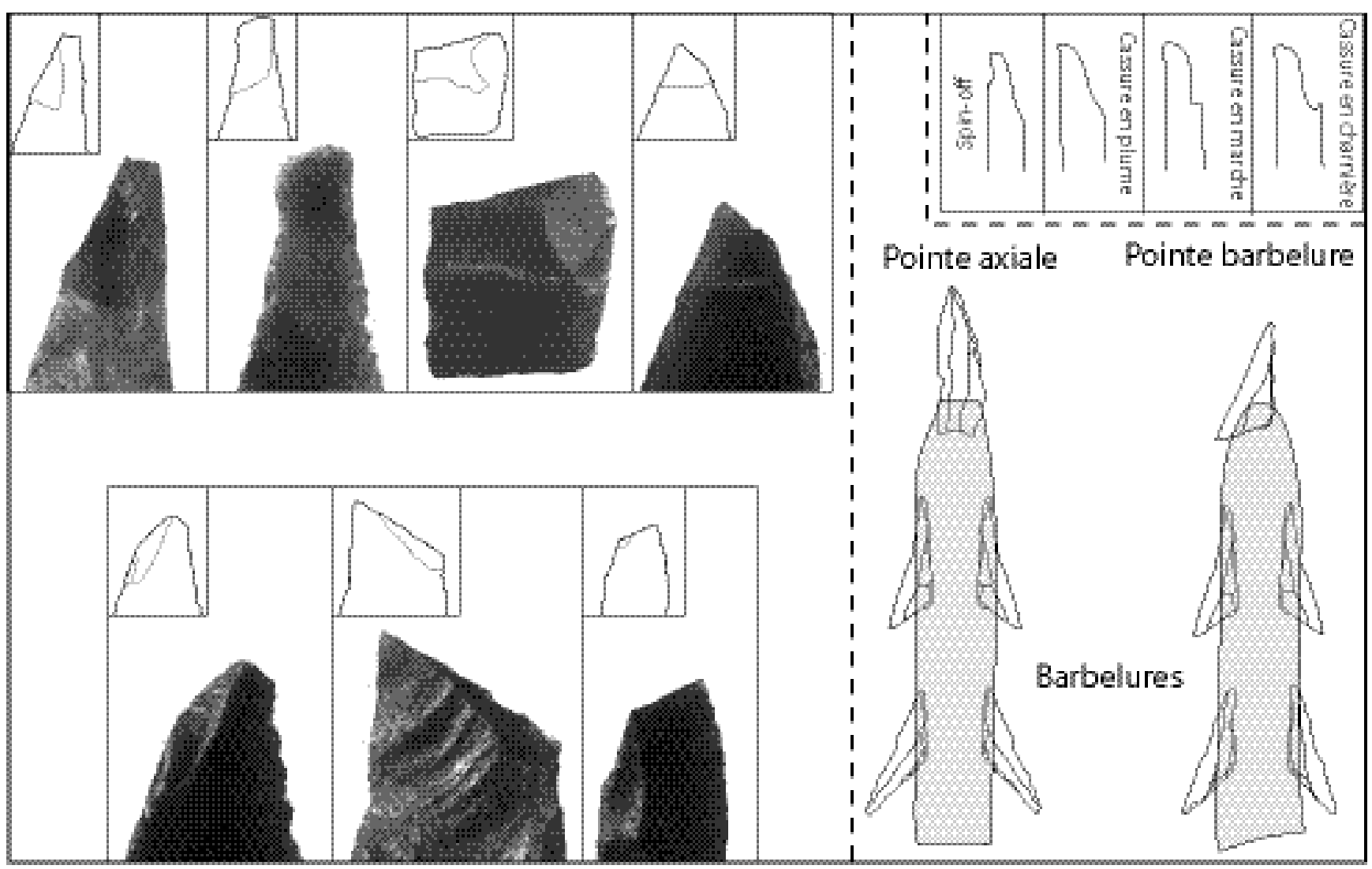

Figure 6 : Armatures : relations entre impacts et emmanchements (d'après Fischer et al., 1984; Philibert, 2002 et Chesnaux, 2008). Figure 6: Armatures: relation between production and fitting.

sont utilisés en tant que supports de l'outillage commun. Les lamelles sont majoritairement extraites sur une table large exploitée de manière semi-tournante à partir d'un plan de frappe incliné vers le dos; un plan de frappe secondaire peut être utilisé pour corriger les convexités du bloc. Différentes options sont ensuite possibles :

- l'exploitation devient périphérique, avec un débitage strictement unipolaire;

- la gestion volumétrique se base sur des changements d'axe de débitage avec, soit une seconde table unipolaire recoupant orthogonalement la première, soit de multiples retournements du bloc.

Ces savoir-faire et choix techniques s'affichent dans d'autres collections du Premier Mésolithique issues de prospections de J. et F. Blanchet, mais aussi à La Pierre-SaintLouis (Geay, Charente-Maritime : Michel, à paraître), à une trentaine de kilomètres de là. On retrouve également les modalités Essart-E, Essart-F, Essart-G telles qu'elles ont été définies sur le site éponyme (Poitiers, Vienne : Marchand (dir.), 2009), auxquelles s'ajoutent des variantes opératoires mais aussi des gestions volumétriques tout autres. Les résultats de l'étude en cours sur la collection E. Patte (1971) de l'abri des Rocs (Bellefonds) permettront de préciser les filiations avec la Vienne. Mais les comparaisons ne s'arrêtent pas là et l'on peut notamment s'en référer à des sites de LoireAtlantique tels que Les Vingt-Deux-Boisselées (Saint-Pèreen-Retz : Tessier, 2000 ; 2001 ; Le Goff, 2002) ou L'Organais (Sainte-Reine-de-Bretagne: Gallais et al., 1985; Le Goff, 2003), même si les options de poursuite du débitage diffèrent alors (Michel, à paraître).

\section{Dans quel territoire stylistique Fontbelle s'inscrit-il?}

Compte tenu de sa position médiane sur la façade atlantique de la France, la Charente-Maritime, au delà même des limites chronologiques du Premier Mésolithique, s'est vue tantôt rattachée à des groupes plus septentrionaux, tantôt soumise à des influences méridionales. Dans le cadre chronologique qui nous intéresse ici, les industries lithiques y ont parfois été attribuées, essentiellement à partir de modèles forgés sur les armatures, à des groupes évoluant en périphérie du centre sauveterrien localisé plus au sud, voire qualifiés de sauveterroïdes. Il est en effet indéniable qu'un lien existe 


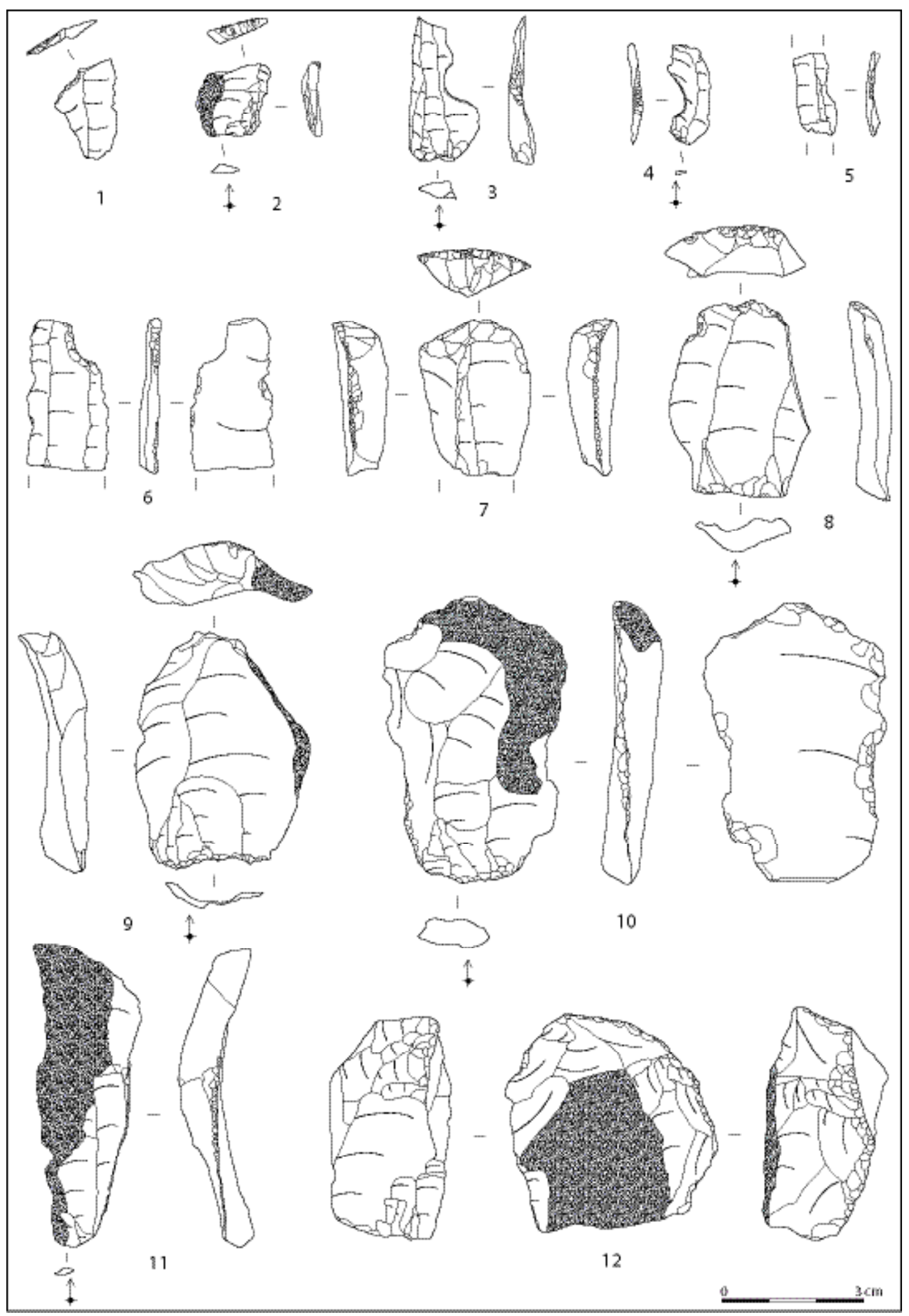

Figure 7 : Outillage commun récolté dans le sondage $1: 1$ et 2, lames tronquées; 3-6, lame(lle)s encochées; 7 et 8 , grattoirs; 9,10 et 12 , éclats retouchés; 11 , lame utilisée.

Figure 7: Common tools from Trench 1: 1 \&, truncated blades; 3-6, notched blade(let)s; 7 \&, scrapers; 9, 10 \& 12, retouched flakes; 11, a posteriori notched blade. 
entre les populations de Charente-Maritime et celles évoluant plus au sud (Gouraud, 1996; 2000; Laporte et al., 2000 ; Marchand et Laporte, 2000). Cependant, deux points peuvent être discutés :

- la notion binaire opposant centre et périphérie, désormais largement contestée car sans doute peu adaptée pour définir des chasseurs-collecteurs;

- la minimisation aussi bien de particularismes locaux que de traits communs aux industries des communautés plus septentrionales ou orientales.

Fontbelle confirme en effet ce qui a été observé dans les Charentes avec un carquois d'une phase ancienne du Mésolithique dominé par des triangles, avec une faible occurrence des triangles de Montclus - qui d'ailleurs sont peu nombreux plus au sud avant le Montclusien (Valdeyron, 1994). Cette préférence stylistique se fait aussi au détriment des pointes, notamment des modèles fusiformes - telles les pointes de Sauveterre, de la Majoire ou de Chaville - absents à Fontbelle, des segments de cercle et des lamelles à bord(s) abattu(s). L'intervention du procédé du microburin dans la fabrication des armatures est fréquente. Par analogie avec le Mésolithique ancien ligérien (Valdeyron, 1994), on pourrait parler pour cette composition typologique d'un "Mésolithique ancien charentais " (Michel, 2007). Ce faciès ne s'intégrerait pas purement et simplement au techno-complexe sauveterrien mais s'insérerait dans de vastes réseaux d'interactions croisées qu'il resterait à définir. Certains auteurs ont soumis l'hypothèse d'un Mésolithique ancien atlantico-ligérien (Roussot-Larroque, 2000; Thévenin, 2005). Le décryptage des triangles de Fontbelle ne va pas à l'encontre de cette idée; en effet, des modèles [a $\mathbf{a}=4-6 \mathrm{~mm}$; $\left.\alpha>115^{\circ}\right]$ et $\left[\mathbf{a}=7-12 \mathrm{~mm} ; \alpha<115^{\circ}\right]$, qui respectivement caractériseraient les industries sauveterriennes ou le Mésolithique ancien ligérien et qui, associées, correspondraient au Mésolithique ancien atlantico-ligérien (Thévenin, 2005), ont été observés à Fontbelle.

\section{4-ConClusions : \\ EN ATTENDANT LES ÉTUDES À VENIR}

Ce site de Fontbelle contribue donc à la construction de modèles sur le Premier Mésolithique en Charente-Maritime. La proportion de triangles isocèles, mais aussi la présence de couteaux à encoches basilaires, plaideraient plus exactement pour des industries de la fin du $9^{\mathrm{e}}$ millénaire avant notre ère. Les chasseurs-collecteurs de Fontbelle ont opté pour l'acquisition de matières siliceuses taillables uniquement locales. La totalité des phases de débitage sont représentées sur le site : à la suite d'une mise en forme simplifiée, le débitage lamellaire est régi par des séquences unipolaires qui peuvent se succéder avec un plan de frappe actif alors différent. La production est intégrée à une unique chaîne opératoire : les sous-produits servent de supports à l'outillage commun alors que les lamelles sont transformées en armatures. Le carquois, dominé par des triangles, traduit, plutôt qu'une zone sous influence méridionale, un " melting-pot » certainement dû à un réseau d'interactions réciproques multidirectionnelles. Si cette série de sondages a permis d'en savoir plus sur l'industrie lithique du Premier Mésolithique, l'organisation du site n'a, en revanche, pas pu être appréhendée compte tenu des dispersions du matériel en milieu sableux. Rappelons pour finir que la récurrence de l'implantation de l'habitat mésolithique sur ce type de terrain résulte localement d'un effet de prospection plutôt que d'une "loi des sables ", comme l'indiquent des occupations voisines en fond de vallée, dont celle de la Grange (Surgères, Charente-Maritime : Laporte et al., 2000; Marchand et Laporte, 2000).

\section{Bibliographie}

Barbaza, M., Valdeyron, N., André, J., Briois, S., Martin, H., Philibert, S., Allios, D. et Lignon, F. 1991 - Fontfaurès en Quercy. Contribution à l'étude du Sauveterrien, Toulouse, École des hautes Études en sciences sociales (Archives d'Écologie préhistorique, 11), $269 \mathrm{p}$.

BARTON, R. N. E., 1987 - Vertical distribution of artefacts and some post-depositional factors affecting site formation, in Rowley-Conwy P., Zvelebil, M. and Blankholm, H. P. (dir.), Mesolithic Northwest Europe: recent trend. University of Sheffield, Department of Archaeology and Prehistory, p. 55-62.

Blanchet, F. et Blanchet, J., 2004 - Les Prises, gisement azilien et mésolithique à Brizambourg (Charente-Maritime). Rapport d'évaluation 2004. Poitiers, Service régional de l'Archéologie de Poitou-Charentes.

Blanchet, F., Blanchet, J., MICHEL, S. et Naudinot, N., 2007 - Les Prises, gisement azilien et mésolithique à Brizambourg (Charente-Maritime). Rapport de fouilles 2007, Poitiers, Service régional de l'Archéologie de Poitou-Charentes.

Chesnaux, L., 2008 - Des microlithes sauveterriens, témoins de l'armement des derniers chasseurs-cueilleurs dans les alpes du Nord, in Pétillon, J.-M., Dias-Meirinho, M.-H., Cattelain, P., Honegger, M., Normand, C. et Valdeyron, N. (coord.), Recherches sur les armatures de projectiles du Paléolithique supérieur au Néolithique (actes du colloque C83, XV congrès de l'UISPP, Lisbonne 2006). Palethnologie, 1, p. 139-153.

Costa, L. J. et Marchand, G., 2006 - Transformation des productions lithiques du Premier au Second Mésolithique en Bretagne et en Irlande, Bulletin de la Société prébistorique française, $103\left(\mathrm{n}^{\circ} 2\right)$, p. 275-290. 
Courtaud, P., Duday, H., Martin, H. et Robin, K., 1999 - La nécropole mésolithique de la Vergne (Charente-Maritime, France), in Bintz, P. et Thévenin, A. (dir.), L'Europe des derniers chasseurs, Epipaléolithique et Mésolithique, Paris, CTHS, p. 287-292.

Crombé, P., 1993 - Tree-fall features on final Palaeolithic and Mesolithic sites situated on sandy solls : how to deal with it, Helinium, 33, p. 50-66.

Cupillard, C. et Richard, A., 1998 - Les derniers chasseurs cueilleurs du massifjurassien et de ses marges (13000-5500 avant Jésus-Christ), Lons-le-Saunier, Centre jurassien du Patrimoine, $229 \mathrm{p}$.

Demars, P.-Y., 1998 - Circulation des silex dans le nord de l'Aquitaine au Paléolithique supérieur, l'occupation de l'espace par les derniers chasseurs-cueilleurs, Gallia-Préhistoire, 40, p. 1-28.

Duday, H. et Courtaud, P., 1998 - La nécropole mésolithique de La Vergne (Charente-Maritime), in Guilaine, J. (dir.), Sépultures d'Occident et genèse des mégalithismes (9000-3500 avant notre ère), Séminaire du Collège de France 1997, Paris, éditions Errance, p. 27-37.

Favre, M., 1993 - Rapport de prospection 1993. Poitiers, Service régional de l'Archéologie de Poitou-Charentes.

Favre, M., 2000 - Rapport de prospection 2000, Poitiers, Service régional de l'Archéologie de Poitou-Charentes.

Fischer, A., Wemming-Hansen, P. et Rasmussen, P., 1984 - Macro and micro wear traces on lithic projectile points: experimental results and prehistoric examples, Journal of Danish archaeology, 3, p. 19-46.

Gallais, J.-Y., Damblon, F., Richard, J., Thiebault, S. et Visset, L., 1985 - Le site à microlithes de l'Organais en Sainte-Reine de Bretagne (Loire-Atlantique), Revue archéologique de l'Ouest, 2, p. 23-45.

Geneste, J.-M., 1992 - L'approvisionnement en matières premières dans les systèmes de production lithique : la dimension spatiale de la technologie, in Mora, R. et Terradas, X. (dir.), Tecnologica y cadenas operativas liticas (reunion international, U.A. Barcelona, 1991), Treballs d'Arqueologia, 1, p. 1-36.

Gouraud, G. 1996 - Le microlithisme de la Pierre-Saint-Louis à Geay (Charente-Maritime) dans le cadre du Mésolithique régional (Mémoire de DEA), Toulouse, université de Toulouse-Le Mirail et École des hautes Études en sciences sociales, 228 p.

Gouraud, G. 2000 - Les microlithes de la zone centrale, couche $5 \mathrm{~B}$, du site de la Pierre-Saint-Louis à Geay (CharenteMaritime), Revue archéologique de l'Ouest, 17, p. 73-86.

Joussaume, R., 1981 - Le Néolithique de l'Aunis et du Poitou occidental dans son cadre atlantique, Rennes, Université de Rennes 1 (Travaux du Laboratoire d'Anthropologie, Préhistoire - Protohistoire et Quaternaire armoricains, 626 p.

Joussaume, R., 1992 - La néolithisation dans le seuil du Poitou et ses marges : milieux karstiques et alluviaux (programme P10 projet 1), rapport 1993, Groupe d'Étude du Néolithique Ancien $d u$ Centre-Ouest, Poitiers, Service régional de l'Archéologie de Poitou-Charentes.

Joussaume, R., 1996 - La néolithisation dans le seuil du Poitou et ses marges : rapport de synthèse 1993-1995, Groupe d'Étude du Néolithique Ancien du Centre-Ouest, Poitiers, Service régional de l'Archéologie de Poitou-Charentes.

Laporte, L., Marchand, G., Sellami, F., Oberlin, C., Bridault, A. et Giraud, T., 2000 - Les occupations mésolithiques et du Néolithique ancien sur le site de la Grange à Surgères (Charente-Maritime), Revue archéologique de l'Ouest, 17, p. 101-142.

Le Goff, G., 2002 - Une étape de l'histoire des techniques: l'étude technologique du locus 1 du site mésolithique ancien des Vingt-Deux-Boisselées, Saint-Père-en-Retz (Loire-Atlantique). (Mémoire de maîtrise), Lorient, Université de Bretagne Sud.

LE Goff, G., 2003 - Le site mésolithique de l'Organais, SainteReine-de-Bretagne (Loire-Atlantique) : étude technologique. (Mémoire de DEA), Rennes, Université de Rennes 2.

Marchand, G., 2008 - Dynamique des changements techniques sur les marges du Massif armoricain de l'Azilien au Premier Mésolithique, in Fagnart, J.-P., Thévenin, A., Ducroce, T., Souffi, B. et Coudret, P. (dir.), Le début du Mésolithique en Europe du nord-ouest (Actes de la table ronde d'Amiens, 2004), Paris, Société préhistorique française, p 52-64.

Marchand, G. et Laporte, L., 2000 - L'habitat mésolithique et néolithique de la Grange à Surgères (Charente-Maritime), in Cupillard, C. (dir.), Les derniers chasseurs-cueilleurs d'Europe occidentale (13000-5500 av. J.-C.) (Actes du colloque international de Besançon, 1998), Besançon, Presses unversitaires Franc-Comtoises (Annales Littéraires, 699; série "Environnement, sociétés et archéologie », 1), p. 253-264.

Marchand, G. (dir.), 2009 - Des feux dans la vallée, les habitats du Mésolithique et du Néolithique récent de l'Essart à Poitiers (Vienne), Rennes, PUR, 246 p.

Michel, S., 2006 - Le premier Mésolithique en Poitou-Charentes et Pays de la Loire : territoires stylistiques et économiques. (Mémoire de Master 2), Université de Rennes 1.

Michel, S., 2007 - Les "territoires " stylistiques du premier Mésolithique en Poitou-Charentes et Pays-de-la-Loire, Revue archéologique de l'Ouest, 24, p. 7-30.

Michel, S., à paraître - Le premier Mésolithique du CentreOuest : modalités techniques de l'industrie lithique.

Patte, E., 1971 - Quelques sépultures du Poitou, du Mésolithique au Bronze moyen, Gallia Préhistoire, 14 (nº 1), p. 139-244.

Pelegrin, J., 2000 - Les techniques de débitage laminaire au Tardiglaciaire : critères de diagnose et quelques réflexions, in Valentin, B., Bodu, P. et Christensen, M. (dir.), L'Europe centrale et septentrionale au Tardiglaciaire (Actes de la table ronde internationale de Nemours, 1997), Nemours, APRAIF (Mémoires du Musée de Préhistoire d’Ile-de-France, 7), p. 73-86. 
Perles, C., 1991 - Économie des matières premières et économie du débitage : deux conceptions opposées?, 25 ans d'études technologiques en Préhistoire : bilan et perspectives, (Actes des $\mathrm{XI}^{\mathrm{e}}$ rencontres internationales d'archéologie et d'histoire d'Antibes, 1990), Juan-les-pins, APDCA, p. 35-45.

Philibert, S., 2002 - Les derniers "sauvages" : territoires économiques et systèmes techno-fonctionnels mésolithiques, Oxford, Hadrian Books (BAR International Series, 1069), 191 p.

Roussot-Larroque, J., 2000 - Sauveterrien et sauveterriens, Préhistoire du Sud-Ouest, 7 (n²), p.159-178.

Rozoy, J.-G., 1978 - Les derniers chasseurs. L'Epipaléolithique en France et en Belgique, Bulletin de la Société archéologique champenoise, $\mathrm{n}^{\circ}$ spécial, $605 \mathrm{p}$.

Tessier, M., 2000 - Le site mésolithique des Vingt-Deux Boisselées $\mathrm{n}^{\circ} 5$ - locus sud à Saint-Père-en-Retz, Groupe vendéen d'Études préhistoriques, $\mathrm{n}^{\circ} 36$, p. 1-6.

Tessier, M., 2001 - Le site mésolithique ancien des 22 Boisselées (locus $\mathrm{n}^{\circ}$ 1). Rapport de prospection-inventaire 2001 dans la zone ouest du Pays de Retz (Loire-Atlantique), Nantes, Service régional de l'Archéologie des Pays de la Loire, p. 47-48.

Thévenin, A., 2005 - L’espace culturel "méditerranéen " : ses expansions de la fin du Tardiglaciaire au début du Postglaciaire, in Jaubert, J. et Barbaza, M. (dir.) - Territoires, déplacements, mobilité, échanges durant la Préhistoire (Actes du 126 congrès national des Sociétés historiques et scientifiques, Toulouse 2001), Paris, CTHS, p.429-451.

Tixier, J. 1963 - Typologie de l'Epipaléolithique du Maghreb. Paris, Mémoires du Centre de Recherches anthropologiques, préhistoriques et ethnographiques Alger, II, 210 p.

Valdeyron, N., 1994 - Le Sauveterrien, culture et sociétés mésolithiques dans la France du Sud durant les $X^{e}$ et IX millénaires $B P$ (Thèse de doctorat), Toulouse, Université de Toulouse-Le Mirail, 584 p.

Vermeersch, P. M., 1976 - La position lithostratigraphique et chronostratigraphique des industries épipaléolithiques et mésolithiques en Basse Belgique. Congrès préhistorique de France, $X X^{e}$ session 1974, Provence. Paris, Société préhistorique française, p. 616-621.

Vermeersch, P. M., 1995 - Processus post-dépositionnels sur des sites épipaléolithiques et mésolithiques en régions sableuses de l'Europe de l'ouest, in Thévenin, A. et Bintz, P. (dir.), L'Europe des derniers chasseurs, peuplement et paléoenvironnement de l'Epipaléolithique et du Mésolithique (Actes du $5^{\mathrm{e}}$ colloque international UISPP, commission 12, Grenoble 1995), Paris, CTHS (Documents préhistoriques, 12), p. 159-166. 
